\title{
STATISTICAL SPECTRAL ANALYSIS OF TIME SERIES ARISING FROM STATIONARY STOCHASTIC PROCESSES ${ }^{1}$
}

\author{
By Ulf Grenander and Murray Rosenblatt
}

\section{University of Chicago}

Summary. We consider time series which are realizations of a stochastic process. From the time series we construct various estimates of the spectral distribution function of the process (Section 3) and we study the sampling distributions of some functionals of these estimates (Sections 4-7). We then obtain confidence regions for the spectral distribution function and various tests of hypotheses in the normal case (Sections 8-10).

1. Introduction. Let us consider a real discrete parameter stochastic process' that is, a sequence of stochastic variables $x_{t}, t=\cdots,-1,0,1, \ldots .^{2} \mathrm{We}$ introduce the quantities $E x_{t}=m_{t}$, the mean value sequence, and the covariances $\rho_{s, t}=E\left(x_{s}-m_{s}\right)\left(x_{t}-m_{t}\right)$. The process $y_{t}=x_{t}-m_{t}$ is said to be stationary in the wide sense if $\rho_{s, t}=\rho_{s-t}$. Then it follows from a theorem of Herglotz [10] that $\rho_{t}=\int_{-\pi}^{\pi} e^{i t \lambda} d F(\lambda)$ where $F(\lambda)$ is a bounded and nondecreasing function in $(-\pi, \pi) . F(\lambda)$ is called the spectral distribution function of the process as it can be said to describe the distribution of the spectral energy of the process (see Wold [22], p. 16). As $x_{t}$ is real, $\rho_{t}=\rho_{-t}$ and the distribution of the spectral energy is symmetric about zero.

Since knowledge of the distribution of the spectral energy is equivalent to knowledge of the covariance sequence, it is a matter of convenience which to choose in analyzing the process. The reduced process $y_{t}$ can be written as

$$
y_{t}=\int_{-\pi}^{\pi} e^{i t \lambda} d Z(\lambda)
$$

[3] where $Z(\lambda)$ is an orthogonal process such that

$$
\begin{aligned}
E Z(\lambda) & =0 \\
E|Z(\lambda)-Z(\mu)|^{2} & =|F(\lambda)-F(\mu)| .
\end{aligned}
$$

(For a discussion of stochastic integrals used in this paper see J. L. Doob, Stochastic Processes, John Wiley and Sons, 1953.) Hence $d F(\lambda)$ can be interpreted as the variance of the stochastic amplitude $d Z(\lambda)$ corresponding to the harmonic $e^{i t \lambda}$ in the Fourier expansion of $y_{t} . F(\lambda)$ seems to be more directly related to the structure of the process than the covariance sequence. Later on

Received 10/22/52.

1 This work has been carried out with support of the Office of Naval Research.

${ }^{2}$ Any notation introduced in the paper will be adhered to in the remainder of the paper without any further explanation. 
we shall find other reasons for preferring to deal with the spectrum instead of the covariances.

We can decompose $F(\lambda)$ into three parts, $F(\lambda)=F_{a}(\lambda)+F_{d}(\lambda)+F_{s}(\lambda)$, where $F_{a}(\lambda)=\int_{-\pi}^{\lambda} f(l) d l, F_{d}(\lambda)=\sum_{\lambda_{\nu} \leqq \lambda} \Delta F\left(\lambda_{v}\right)$, and $F_{s}(\lambda)$ is constant except on a set of Lebesgue measure zero. Here $f(\lambda)=F^{\prime}(\lambda)$, the spectral density, is a nonnegative integrable function and the $\lambda_{\nu}$ 's are the points of discontinuity of $F(\lambda)$ with associated jumps $\Delta F\left(\lambda_{\nu}\right)>0$. We are going to deal with the absolutely continuous case, but will make a few remarks later on concerning possible discontinuities of the spectral distribution function.

A model of some practical interest is the following one. A linear filter $\mathcal{F}$ can be characterized by its output $a_{t}$ at time $t$ generated by a unit input at time 0 . It is not necessary for our purpose to demand that $a_{t}=0$ for $t<0$. Suppose we feed independent random impulses $\xi_{t}$, identically distributed with mean 0 and standard deviation 1, into the filter. Following Tukey we shall call a process of the type $\xi_{t}$ "pure white noise" as contrasted with a more general process $\eta_{t}$ with $E \eta_{t}=0, E \eta_{s} \eta_{t}=\delta_{s t}$ (called "white noise"), but where the $\eta^{\prime}$ 's are not necessarily independent. Note that in both cases one has a uniform spectrum, $F^{\prime}(\lambda)=(2 \pi)^{-1}$. The resulting output at time $t$ is then

$$
y_{t}=\sum_{n=-\infty}^{\infty} a_{n} \xi_{t-n} .
$$

If $\sum_{-\infty}^{\infty} a_{v}^{2}<\infty$, this sum converges with probability 1 (see Levy [15], p. 139142). If the input had been $\eta_{t}$ we would only be able to state convergence in the mean square. In both cases the spectrum is absolutely continuous with spectral density (see Karhunen [12], p. 71)

$$
f(\lambda)=\frac{1}{2 \pi}\left|\sum_{n=-\infty}^{\infty} a_{n} e^{-i n \lambda}\right|^{2}
$$

More restrictive conditions will be imposed on the process later. A process of the type (1.3) is called a linear process.

On the other hand, if a real stationary process $y_{t}$ has an absolutely continuous spectral distribution function $F(\lambda)=\int_{-\pi}^{\lambda} f(l) d l$, Doob (see Proceedings of the Berkeley Symposium on Mathematical Statistics and Probability, University of California Press, 1949, p. 327) has shown that

$$
y_{t}=\sum_{n=-\infty}^{\infty} a_{n} \eta_{t-n},
$$

where we can choose $a_{n}=\int_{-\pi}^{\pi} e^{i n \lambda} \sqrt{f(\lambda)} d \lambda$ and $E \eta_{t}=0, E \eta_{s} \eta_{t}=\delta_{s}$. The $\eta$ 's are elements of the real Hilbert space spanned by the $y$ 's and an appropriately chosen set of real random variables (see [12], p. 42). They are then real and so are the $a$ 's. In the present paper we shall deal only with the linear proc- 
esses. It would be interesting to extend the domain in which our results are valid to a more general class of processes.

A normally distributed process with an absolutely continuous spectrum is clearly linear as the $\eta$ 's appearing in (1.4) are then automatically independent and identically distributed.

2. Some methods that have been proposed for time series analysis. An important group of statistical problems arise in the following way. We observe a sample $x_{1}, x_{2}, \cdots, x_{N}$ (a time series) from the stochastic process $x_{t}$ and want to make inferential statements concerning the covariances, or equivalently the spectrum of $x_{t}$. There has been a long history of work aimed at answering such problems. A considerable part of the literature has been devoted to testing the independence of the observations, usually in the case of normal time series (for a list of references see [14]). Other statisticians have studied what one could call finite parameter schemes. Two important schemes of this type are the autoregressive scheme (2.1) and the moving average (2.2)

$$
\begin{aligned}
& \sum_{n=0}^{p} b_{n} y_{t-n}=\xi_{t} \\
& y_{t}=\sum_{n=0}^{p} \beta_{n} \xi_{t-n}
\end{aligned}
$$

where the $b$ 's and the $\beta$ 's are constants. Specifying a priori an order $p$ for the process, they have given estimates for the coefficients and have devised tests for various hypotheses (for a list of references see [14]). Whittle [21] has gone further and has given tests for discriminating between finite parameter schemes of different orders without specifying the coefficients a priori.

Any linear process can be approximated as closely as is desired by a finite parameter scheme of sufficiently high order. In most practical situations, however, it does not seem realistic to take the order of the scheme as some number $p$, usually small, given a priori.

A great deal of the older literature has been devoted to the so-called periodogram analysis (see Kendall [13] for references). This was originally devised to deal with processes of the type

$$
x_{t}=\sum_{\nu=1}^{m}\left(A_{\nu} \sin \lambda_{\nu} t+B_{\nu} \cos \lambda_{\nu} t\right)+\xi_{t}
$$

where $m, A_{\nu}, B_{\nu}$, and $\lambda_{\nu}$ are unknown. Clearly $x_{t}$ has a spectrum with a discrete component. To estimate the frequencies $\lambda_{\nu}$ the following statistic called the periodogram

$$
\begin{aligned}
I_{N}(\lambda) & =\frac{1}{2 \pi N}\left|\sum_{\nu=1}^{N} x_{\nu} e^{-i \nu \lambda}\right|^{2} \\
& =\frac{1}{2 \pi N}\left[\left(\sum_{\nu=1}^{N} x_{\nu} \cos \nu \lambda\right)^{2}+\left(\sum_{\nu=1}^{N} x_{\nu} \sin \nu \lambda\right)^{2}\right]
\end{aligned}
$$


has been used. A rationale for this method is that $I_{N}(\lambda)$ can be shown to diverge to infinity as $N \rightarrow \infty$ if $\lambda$ coincides with some frequency $\lambda_{\nu}$. Hence if $I_{N}(\lambda)$ is large we suspect that $\lambda$ is one of the frequencies $\lambda_{\nu}$ of the scheme. For a corresponding test of significance see Fisher [6].

Periodogram analysis is not immediately applicable to the case of an absolutely continuous spectrum. However, some work of the last few years has indicated that when it is properly modified, it is the most powerful method that has been found to work without very special assumptions concerning the covariance structure of the process. We would like to mention especially Bartlett [1], [2], and Tukey [20].

A brief discussion of some of the results of this paper can be found in [9].

3. Some preliminary considerations. Consider a process

$$
y_{t}=\int_{-\pi}^{\pi} e^{-i t \lambda} d Z(\lambda)=\sum_{\nu} Z_{\nu} e^{-i t \lambda}+y_{t}^{\prime}
$$

where $y_{t}^{\prime}$ is the component of the process with a continuous spectrum and the other term is the one with a discrete spectrum. If we observe a complete realization $y_{t},-\infty<t<\infty$, we can specify the sample value of any $Z_{\nu}$ with probability one. However, we cannot estimate $E\left|Z_{\nu}\right|^{2}=\Delta F\left(\lambda_{\nu}\right)$ consistently unless $\left|Z_{\nu}\right|$ is constant with probability one. We note in passing that the model of random phases in which $Z_{\nu}=A_{\nu} e^{i \varphi_{\nu}}$, where the $\varphi_{\nu}$ 's are independent and uniformly distributed in $(-\pi, \pi)$ and the $A_{\nu}$ 's are constants, has this property and is not without interest (see Lêvy [16], p. 114).

Although the periodogram is a legitimate tool for estimating the frequencies $\lambda_{\nu}$ of a discrete spectrum as has been remarked above, it cannot be used to estimate the spectral density of an absolutely continuous spectrum consistently [7]. Still the periodogram plays a fundamental role in our paper as our estimates are closely related to it.

Let $y_{t}$ be a linear process and let the $\xi$ 's used in constructing the process have a fourth moment $\mu_{4}$. We set

$$
e=\mu_{4}-3 .
$$

Note that $e$ is the fourth cumulant of the random variables $\xi$. The periodogram $I_{N}(\lambda)$ of the process $y_{t}$ then is

$$
\begin{aligned}
I_{N}(\lambda) & =\frac{1}{2 \pi N}\left|\sum_{t=1}^{N} y_{t} e^{-i t \lambda}\right|^{2} \\
& =\frac{C_{0}}{2 \pi N}+\frac{1}{\pi N} \sum_{\nu=1}^{N-1} C_{\nu} \cos \nu \lambda
\end{aligned}
$$

where $C_{\nu}=\sum_{t=1}^{N-\nu} y_{t} y_{t+\nu}$. We are interested in statistics of the form

$$
\Phi_{N}=\int_{-\pi}^{\pi} I_{N}(l) \phi(l) d l
$$


where $\phi(l)$ is bounded, symmetric about zero, and has at most a finite number of discontinuities. Let

$$
\Phi=\int_{-\pi}^{\pi} f(l) \phi(l) d l
$$

Theorem 1. Let $\Phi_{N}, \Phi$ be defined as in (3.1), (3.2) and let $\Psi_{N}, \Psi$ be defined analogously with weight function $\psi$. If the spectral density $f(l)$ is continuous

and

$$
\lim _{N \rightarrow \infty} E \Phi_{N}=\Phi, \quad \lim _{N \rightarrow \infty} E \Psi_{N}=\Psi
$$

$$
\lim _{N \rightarrow \infty} N \operatorname{cov}\left(\Phi_{N}, \Psi_{N}\right)=e \Phi \Psi+4 \pi \int_{-\pi}^{\pi} f^{2}(l) \psi(l) \phi(l) d l .
$$

Proof. By definition of $\Phi_{N}$

$$
E \Phi_{N}=\int_{-\pi}^{\pi} E I_{N}(l) \phi(l) d l .
$$

But using (1.1)

$$
I_{N}(l)=\frac{1}{2 \pi N}\left|\sum_{1}^{N} y_{\nu} e^{-i \nu l}\right|^{2}=\frac{1}{2 \pi N}\left|\int_{-\pi}^{\pi} e^{-i(\lambda-l)} \frac{1-e^{i N(\lambda-l)}}{1-e^{i(\lambda-l)}} d Z(\lambda)\right|^{2}
$$

and hence from (1.2)

$$
\begin{aligned}
E I_{N}(l) & =\frac{1}{2 \pi N} \int_{-\pi}^{\pi}\left|e^{i(\lambda-l)} \frac{1-e^{i N(\lambda-l)}}{1-e^{i(\lambda-l)}}\right|^{2} d E|Z(\lambda)|^{2} \\
& =\frac{1}{2 \pi N} \int_{-\pi}^{\pi} \frac{\sin ^{2} \frac{N}{2}(\lambda-l)}{\sin ^{2} \frac{\lambda-l}{2}} f(\lambda) d \lambda
\end{aligned}
$$

which tends to $f(l)$ as $N \rightarrow \infty$. But the integrand in (3.4) is bounded by

$$
|\phi(\lambda)| \max _{\lambda} f(\lambda)
$$

so that we have $E \Phi_{N} \rightarrow \Phi$ as $N \rightarrow \infty$.

Let us now consider $N \operatorname{cov}\left(\Phi_{N}, \Psi_{N}\right)$. We have

$$
N \operatorname{cov}\left(I_{N}(\lambda), I_{N}(\mu)\right)=\frac{1}{4 \pi^{2} N} \sum_{\alpha, \beta, \gamma, \delta=1}^{N} \operatorname{cov}\left(y_{\alpha} y_{\beta}, y_{\gamma} y_{\delta}\right) e^{i \lambda(\alpha-\beta)-i \mu(\gamma-\delta)} .
$$

But

$$
\begin{aligned}
E y_{\alpha} y_{\beta} y_{\gamma} y_{\delta} & =\sum_{\nu_{1}, p_{2}, \nu_{3}, \nu_{4}=-\infty}^{\infty} a_{\alpha-\nu_{1}} a_{\beta-\nu_{2}} a_{\gamma-\nu_{3}} a_{\delta-\nu_{4}} E \xi_{\nu_{1}} \xi_{\nu_{2}} \xi_{\nu_{3}} \xi_{\nu_{4}} \\
& =\left(\mu_{4}-3\right) \sum_{\nu=-\infty}^{\infty} a_{\alpha-\nu} a_{\beta-\nu} a_{\gamma-\nu} a_{\delta-\nu}+\sum_{\nu, \mu=-\infty}^{\infty} a_{\alpha-\nu} a_{\beta-\nu} a_{\gamma-\mu} a_{\delta-\mu} \\
& +\sum_{, \mu=-\infty}^{\infty} a_{\alpha-\nu} a_{\beta-\mu} a_{\gamma-\mu} a_{\delta-\nu}+\sum_{\nu, \mu=-\infty}^{\infty} a_{\alpha-\nu} a_{\beta-\mu} a_{\gamma-\nu} a_{\delta-\mu}
\end{aligned}
$$


so that

$$
\operatorname{cov}\left(y_{\alpha} y_{\beta}, y_{\gamma} y_{\delta}\right)=\left(\mu_{4}-3\right) \sum_{\nu=-\infty}^{\infty} a_{\alpha-\nu} a_{\beta-\nu} a_{\gamma-\nu} a_{\delta-\nu}+\rho_{\alpha-\delta} \rho_{\beta-\gamma}+\rho_{\alpha-\gamma} \rho_{\beta-\delta} .
$$

The fourth moments of $Y_{\alpha}$ can be shown to exist and the operations that have been carried out can be justified by a repeated application of Schwarz' inequality and Fatou's lemma. Hence

$N \operatorname{cov}\left(\Phi_{N}, \Psi_{N}\right)=\frac{1}{4 \pi^{2} N}$

$$
\begin{array}{r}
\left\{\sum_{\alpha, \beta, \gamma, \delta=1}^{N}\left[\left(\mu_{4}-3\right) \sum_{\nu=-\infty}^{\infty} a_{\alpha-\nu} a_{\beta-\nu} a_{\gamma-\nu} a_{\delta-\nu}+\rho_{\alpha-\delta} \rho_{\beta-\gamma}+\rho_{\alpha-\gamma} \rho_{\beta-\delta}\right] \phi_{\alpha-\beta} \bar{\psi}_{\gamma-\delta}\right\} \\
=S_{1}+S_{2}+S_{3}
\end{array}
$$

where $\phi_{\nu}=\int_{-\pi}^{\pi} e^{i \nu \lambda} \phi(\lambda) d \lambda$ and $\psi_{\nu}$ is defined analogously.

We first deal with $S_{1}$. Note that

$$
\begin{aligned}
S_{1} & =\frac{\mu_{4}-3}{4 \pi^{2} N} \int_{-\pi}^{\pi} \int_{-\pi}^{\pi} \sum_{\nu=-\infty}^{\infty} \sum_{\alpha, \beta=1}^{N} a_{\alpha-\nu} a_{\beta-\nu} e^{i(\alpha-\beta) \lambda} \sum_{\gamma, \delta=1}^{N} a_{\gamma-\nu} a_{\delta-\nu} e^{-i(\gamma-\delta) \mu} \phi(\lambda) \psi(\mu) d \lambda d \mu \\
& =\frac{\mu_{4}-3}{4 \pi^{2} N} \int_{-\pi}^{\pi} \int_{-\pi}^{\pi} \sum_{\nu=-\infty}^{\infty}\left|\sum_{\alpha=1}^{N} a_{\alpha-\nu} e^{i \alpha \lambda}\right|^{2}\left|\sum_{\gamma=1}^{N} a_{\gamma-\nu} e^{i \gamma \mu}\right|^{2} \phi(\lambda) \psi(\mu) d \lambda d \mu .
\end{aligned}
$$

First we consider the special case

The function

$$
\phi(\lambda)=e^{-i n \lambda}, \quad \psi(\mu)=e^{-i n \mu} .
$$

$$
c_{n, m}(\lambda)=\sum_{k=-\infty}^{\infty} a_{k} a_{k-n} e^{i k \lambda} \sum_{l=-\infty}^{\infty} a_{l} a_{l-m} e^{-i l \lambda}
$$

is continuous. We have

$$
\frac{1}{2 \pi} \int_{-\pi}^{\pi} c_{n, m}(\lambda) e^{-i(\alpha-\gamma) \lambda} d \lambda=\sum_{\nu=-\infty}^{\infty} a_{\alpha-\nu} \alpha_{\alpha-n-\nu} a_{\gamma-\nu} a_{\gamma-m-\nu}
$$

so that

$$
S_{1}\left(e^{-i n \lambda} e^{-i m \mu}\right)=\frac{e}{4 \pi^{2} N}(2 \pi)^{2} \sum_{\alpha=\max (1,1+m)}^{\min (N, N+m)} \sum_{\gamma=\max (1,1+n)}^{\min (N, N+n)} \frac{1}{2 \pi} \int_{-\pi}^{\pi} c_{n, m}(\lambda) e^{-i(\alpha-\gamma) \lambda} d \lambda .
$$

But then

$$
\begin{aligned}
S_{1}\left(e^{-i n \lambda} e^{-\imath m \mu}\right)= & \frac{e}{2 \pi N} \int_{-\pi}^{\pi} e^{\frac{i(n-m) \lambda}{2}} c_{n, m}(\lambda) \frac{\sin \frac{N-m}{2} \lambda \sin \frac{N-n}{2} \lambda}{\sin ^{2} \frac{\lambda}{2}} d \lambda \\
=\frac{e}{2 \pi N} \int_{-\pi}^{\pi} e^{\frac{i(n-m) \lambda}{2}} c_{n, m}(\lambda) & {\left[\frac{\sin ^{2} \frac{N-n}{2} \lambda}{\sin ^{2} \frac{\lambda}{2}} \cos \frac{n-m}{2} \lambda+\frac{1}{2} \frac{\sin (N-n) \lambda \sin \frac{n-m}{2} \lambda}{\sin ^{2} \frac{\lambda}{2}}\right] d \lambda . }
\end{aligned}
$$


Using [5] we see that the second term is dominated by constant $(\log N) /(N) \rightarrow 0$ as $N \rightarrow \infty$. The first term tends to $e c_{n, m}(0)=e \rho_{n} \rho_{m}$. Now let us consider a finite trigonometric polynomial

$$
h(\lambda, \mu)=\sum_{n, m=-p}^{p} h_{n, m} e^{-i n \lambda} e^{-i m \mu}
$$

in place of $\phi(\lambda) \psi(\lambda)$ in $S_{1}$. Then

$$
\lim _{N \rightarrow \infty} S_{1}(h(\lambda, \mu))=\frac{e}{4 \pi^{2}} \sum_{n, m=-p}^{p} h_{n, m} \rho_{n} \rho_{m}=e \int_{0}^{\pi} \int_{0}^{\pi} h(\lambda, \mu) f(\lambda) f(\mu) d \lambda d \mu .
$$

Now given any $\phi(\lambda)$ and $\psi(\mu)$, we can choose two finite trigonometric polynomials $h_{1}(\lambda, \mu), h_{2}(\lambda, \mu)$ such that

$$
h_{1}(\lambda, \mu) \leqq \phi(\lambda) \psi(\mu) \leqq h_{2}(\lambda, \mu), \quad \int_{-\pi}^{\pi} \int_{-\pi}^{\pi}\left[h_{2}(\lambda, \mu)-h_{1}(\lambda, \mu)\right] d \lambda d \mu<\epsilon .
$$

Then $S_{1}(\phi(\lambda) \psi(\mu))$ lies between $S_{1}\left(h_{1}(\lambda, \mu)\right), S_{1}\left(h_{2}(\lambda, \mu)\right)$ and hence

$$
\varlimsup_{N \rightarrow \infty}, \lim _{N \rightarrow \infty} S_{1}(\phi(\lambda) \psi(\mu))
$$

lies between $e !_{0}^{\pi} \int_{0}^{\pi} h_{1}(\lambda, \mu) f(\lambda) f(\mu) d \lambda d \mu$ and $e \int_{0}^{\pi} !_{0}^{\pi} h_{2}(\lambda, \mu) f(\lambda) f(\mu) d \lambda d \mu$. On letting $\epsilon \rightarrow 0$ we see that

$$
\lim _{N \rightarrow \infty} S_{1}(\phi(\lambda) \psi(\mu))=e \int_{0}^{\pi} \phi(\lambda) f(\lambda) d \lambda \int_{0}^{\pi} \psi(\mu) f(\mu) d \mu .
$$

To evaluate the remainder $S_{2}+S_{3}$ of $\operatorname{cov}\left(\Phi_{N}, \Psi_{N}\right)$ we note that it would be the actual covariance if the process were normal. But in this case the limiting covariance has been evaluated in [7] when $\phi$ and $\psi$ are even, although under the restriction that the spectral density has a continuous derivative. However, an argument similar to that just carried out above indicates that the result in [7] is valid for continuous $f(\lambda)$. This proves (3.3).

One should note that as the periodogram is an even function, it is sufficient to consider estimates of the form $\Phi_{N}=\int_{0}^{\pi} I_{N}(\lambda) \phi(\lambda) d \lambda$ corresponding to the weight function $\frac{1}{2}[\phi(\lambda)+\phi(-\lambda)]$. Expression (3.3) then becomes

$$
\lim _{N \rightarrow \infty} N \operatorname{cov}\left(\Phi_{y}, \Psi_{N}\right)=e \Phi \Psi+2 \pi \int_{0}^{\pi} f^{2}(l) \phi(l) \psi(l) d l .
$$

The following theorem gives an estimate of the speed with which $E \Phi_{v}$ converges to $\Phi$.

Theorem 2. Suppose that $f(\lambda)$ has a bounded derivative. Then

$$
E \Phi_{N}=\Phi+0(\log N / N) \text {. }
$$

Proof. By the proof of Theorem 1

$$
E \Phi_{N}-\Phi=\int_{-\pi}^{\pi} \frac{1}{2 \pi N} \int_{-\pi}^{\pi} \frac{\sin ^{2} \frac{N}{2}(\lambda-l)}{\sin ^{2} \frac{\lambda-l}{2}}[f(l)-f(\lambda)] \phi(\lambda) d \lambda d l .
$$


But we have uniformly $f(l)-f(\lambda)=O(l-\lambda)$ so that

$$
E \Phi_{N}-\Phi=\frac{1}{2 \pi N} \int_{-2 \pi}^{2 \pi} \frac{\sin ^{2} \frac{N}{2} x}{\sin ^{2} \frac{x}{2}} O(|x|) \sqrt{2}(2 \pi-|x|) d x \leqq \frac{K \log N}{N}
$$

according to [5].

We remark that if the function $f(\lambda)$ defined on the real line with period $2 \pi$ has a bounded second derivative everywhere we get instead $E \Phi_{N}-\Phi=O(1 / N)$.

We now prove three elementary lemmas that are required in the development of the main results of the paper. These results have been proved under conditions that are probably far from necessary. But we believe these conditions give an idea of the practical domain of applicability of the results.

In the remainder of the paper we shall assume that $E\left|\xi_{\nu}\right|^{8}<\infty$.

Lemma 1. Consider the covariances $c_{\nu}=\sum_{n=1}^{N-|\nu|} \xi_{n} \xi_{n+|\nu|}$. Then if $\nu, \mu \neq 0$

$$
\left|E c_{\nu} c_{\nu+j} c_{\mu} c_{\mu+j}\right| \leqq\left\{\begin{array}{lll}
A_{1} N & \text { if } & \mu \neq \nu \\
A_{2} N^{2} & \text { if } & \mu=\nu .
\end{array}\right.
$$

The reader should notice that $C_{\nu}$ refers to the $y$-process and $c_{\nu}$ to the $\xi$-process.

Proof. It is clear that (3.5) is made up of terms of the type $E \prod_{i=1}^{8} \xi_{n_{1}}$. If one of the indices $n_{i}$ is different from the rest this term vanishes. As each of the terms is bounded by $E\left|\xi_{\nu}\right|^{8}$, it is sufficient for our purpose to enumerate the nonvanishing terms. But we have restraints, say

$$
n_{2}=n_{1}+a, \quad n_{4}=n_{3}+b, \quad n_{6}=n_{5}+c, \quad n_{8}=n_{7}+d,
$$

where we do not yet specify the integers $a, b, c, d$ except that they should all be different from zero. We can then treat the eight variables in a completely symmetric way. Let us fix $n_{1}$. As $n_{2} \neq n_{1}, n_{2}$ has to be equal to some other $n_{i}$, say $n_{3}$.

Now we separate two cases. As $n_{5} \neq n_{6}$, it has to be equal to one of $n_{1}, n_{2}$, $n_{3}, n_{4}$ or to one of $n_{7}, n_{3}$. Let us consider the first alternative. Then $n_{7}$ has to be equal to one of $n_{1}, n_{2}, n_{3}, n_{4}, n_{5}, n_{6}$ and whichever we choose, we have

$$
n_{i}=n_{1}+\alpha_{i}, \quad i=2,3, \cdots, 8 .
$$

As $1 \leqq n_{i} \leqq N$ this gives us at most $N$ possibilities.

In the second alternative let us take $n_{5}=n_{7}$. If any of $n_{1}, n_{2}, n_{3}, n_{4}$ is equal to any of $n_{5}, n_{6}, n_{7}, n_{8}$ we have again a system of restrictions of type (3.6) and hence again at most $N$ possibilities so we can exclude this case. But $n_{2}=n_{3} \neq n_{1}$ and $n_{6} \neq n_{7}=n_{5}$ so that the only other way of getting a nonvanishing term is $n_{2}=n_{4}, n_{6}=n_{8}$, which requires that $a=b, c=d$. If that is the case we clearly get at most $N^{2}$ possibilities. The result now follows easily.

Lemma 2. The distribution of the variables $\left(c_{0}-N\right) / \sqrt{\bar{N}}, c_{1} / \sqrt{\bar{N}}, \cdots, c_{k} / \sqrt{\bar{N}}$ tends to the distribution of $k+1$ normal and independent variables with mean zero and variances $e+2,1,1, \cdots, 1$. 
Proof. Introduce

$$
z_{j}=t_{0}\left(\xi_{j}^{2}-1\right)+t_{1} \xi_{j} \xi_{j+1}+\cdots+t_{k} \xi_{j} \xi_{j+k}
$$

so that

$$
U_{N}=\frac{1}{\sqrt{\bar{N}}} \sum_{j=1}^{\mathrm{v}} z_{j}=\frac{1}{\sqrt{\bar{N}}}\left[t_{0}\left(c_{0}-N\right)+t_{1} c_{1}^{\prime}+\cdots+t_{k} c_{k}^{\prime}\right]
$$

where

$$
c_{\nu}^{\prime}=c_{\nu}+\sum_{j=N-\nu+1}^{N} \xi_{j} \xi_{j+\nu}, \quad \nu>0 .
$$

It is evident that $\left(c_{\nu}^{\prime}-c_{\nu}\right) /(\sqrt{N}) \rightarrow 0$ in probability as $N \rightarrow \infty$. But $z_{j}$ is a stationary $(k+1)$-dependent sequence of random variables. Applying a theorem of Hoeffding and Robbins [11] we see that $U_{N}$ is asymptotically normal with mean zero and variance $t_{0}^{2}(e+2)+\sum_{1}^{k} t_{\nu}^{2}$. Hence

$$
E e^{i U_{N}}=E \exp \left\{i t_{0} \frac{c_{0}-N}{\sqrt{\bar{N}}}+i \sum_{1}^{k} t_{\nu} \frac{c_{\nu}^{\prime}}{\sqrt{\bar{N}}}\right\} \rightarrow e^{-\frac{1}{2}\left[t_{0}^{2}(e+2)+\Sigma_{1}^{k} t_{\nu}^{2}\right]}
$$

which proves the lemma.

Lemma 3. If $y_{t}$ is a normal process with a positive spectral density having an integrable second derivative, then $a_{\nu}$ can be chosen so that $a_{\nu}=O\left(1 / \nu^{2}\right)$.

Proof. Choose $a_{\nu}$ so that $a_{\nu}=\int_{-\pi}^{\pi} e^{i \nu \lambda} \sqrt{f(\lambda)} d \lambda$ and integrate by parts twice. The result follows immediately.

\section{Treatment of pure white noise.}

THEOREM 3. Consider the empirical spectral distribution function

$$
F_{N, \xi}^{*}(\lambda)=\int_{0}^{\lambda} I_{N, \xi}(l) d l
$$

where

$$
I_{N, \xi}(\lambda)=\frac{1}{2 \pi N}\left|\sum_{\nu=1}^{N} \xi_{\nu} e^{-i \nu \lambda}\right|^{2} .
$$

Then the limiting probability distribution of

$$
\max _{0 \leqq \lambda \leqq \pi} \sqrt{N}\left|F_{N, \xi}^{*}(\lambda)-\frac{\lambda}{2 \pi}\right|
$$

as $N \rightarrow \infty$ is the same as that of

$$
\max _{0 \leqq \lambda \leqq \pi}|\zeta(\lambda)|
$$

where $\zeta(\lambda)$ is a normally distributed process with mean zero and covariance

$$
E \zeta(\lambda) \zeta(\mu)=\frac{e \lambda \mu}{4 \pi^{2}}+\frac{1}{2 \pi} \min (\lambda, \mu) .
$$


Proof. We have

$$
\begin{aligned}
\sqrt{N}\left[F_{N, \xi}^{*}(\lambda)-\frac{\lambda}{2 \pi}\right]=\frac{\lambda}{2 \pi}\left(\frac{c_{0}}{\sqrt{N}}-\sqrt{\bar{N}}\right)+\sum_{1}^{N-1} \frac{c_{\nu}}{\sqrt{\bar{N}}} \frac{\sin \nu \lambda}{\pi \nu} \\
=s_{N, k}(\lambda)+r_{N, k}(\lambda)
\end{aligned}
$$

where $s_{N, k}(\lambda)$ consists of the first term and the $k-1$ first summands of the sum. We shall show that with probability close to one, for sufficiently large $k$, $\left|r_{N, k}(\lambda)\right|$ is small uniformly in $N, \lambda$.

Let us consider

$$
\left|\sum_{\nu=m}^{l} \frac{c_{\nu}}{\sqrt{\bar{N}}} \frac{\sin \nu \lambda}{\nu}\right| \leqq\left|\sum_{\nu=m}^{l} \frac{c_{\nu}}{\sqrt{\bar{N}}} \frac{e^{i \nu \lambda}}{\nu}\right|
$$

where $0<m<l<N$. But

$$
\left|\sum_{\nu=m}^{l} \frac{c_{\nu}}{\sqrt{\bar{N}}} \frac{e^{i \nu \lambda}}{\nu}\right|^{2} \leqq 2 \sum_{j=0}^{l-m}\left|\sum_{\nu=m}^{l-j} \frac{c_{\nu} c_{\nu+j}}{N \nu(\nu+j)}\right| .
$$

To get a bound for this sum we consider

$$
E\left|\sum_{\nu=m}^{l-j} \frac{c_{\nu} c_{\nu+j}}{N \nu(\nu+j)}\right|^{2}=\sum_{\nu, \mu=m}^{l-j} \frac{E c_{\nu} c_{\nu+j} c_{\mu} c_{\mu+j}}{N^{2} \nu(\nu+j) \mu(\mu+j)} .
$$

We know by Lemma 1 that

$$
\left|E c_{\nu} c_{\nu+j} c_{\mu} c_{\mu+j}\right| \leqq\left\{\begin{array}{lll}
A_{2} N^{2} & \text { if } & \nu=\mu \\
A_{1} N & \text { if } & \nu \neq \mu .
\end{array}\right.
$$

Thus the expression in (4.1) is bounded (use the Schwarz inequality) by

$$
A_{2} \sum_{\nu=m}^{l-j} \frac{1}{\nu^{2}(\nu+j)^{2}}+\frac{A_{1}}{N} \sum_{\nu, \mu=m}^{l-j} \frac{1}{\nu(\nu+j) \mu(\mu+j)} \leqq \sum_{\nu=m}^{l-j} \frac{A_{3}}{\nu^{2}(\nu+j)^{2}} .
$$

Now we choose $m=2^{p}$ and $l=2^{p+1}$. Then again using the Schwarz inequality we get

$$
\begin{aligned}
E \max _{0 \leqq \lambda \leqq \pi}\left|\sum_{\nu=2 p}^{2 p+1} \frac{c_{\nu}}{\sqrt{\bar{N}}} \frac{\sin \nu \lambda}{\nu}\right| \leqq A_{4} \sum_{j=0}^{2 p}\left[\sum_{\nu=2^{p}}^{2^{p+1}-j} \frac{1}{\nu^{2}(\nu+j)^{2}}\right]^{1 / 2} & \\
& \leqq A_{5} \sum_{j=0}^{2 p} \frac{1}{2^{3 p / 2}}=A_{5} / 2^{p / 2}
\end{aligned}
$$

Let $k=2^{n}$. Then with probability greater than $1-A_{5} / 2^{p / 4}$

$$
T_{p}=\max _{0 \leqq \lambda \leqq \pi}\left|\sum_{\nu=2^{p}}^{2 p+1} \frac{c_{\nu}}{\sqrt{\bar{N}}} \frac{\sin \nu \lambda}{\nu}\right|<\frac{1}{2^{p / 4}} .
$$

Consequently

$$
\left|r_{N, k}(\lambda)\right| \leqq \sum_{p=n}^{[\log N]+1} T_{p} \leqq A_{6} \frac{1}{2^{n / 4}}
$$


with probability greater than $1-A_{6} / 2^{n / 4}$. Choosing $n$ sufficiently large $\left|r_{N, k}(\lambda)\right|$ is uniformly small.

We want to find the limiting value of

$$
P\left(\max _{0 \leqq \lambda \leqq \pi} \sqrt{N}\left|F_{N, \xi}^{*}(\lambda)-\frac{\lambda}{2 \pi}\right| \leqq \alpha\right) .
$$

But from what we have just shown, we see that for any $\epsilon, \delta>0$

$$
\begin{aligned}
P\left(\max _{0 \leqq \lambda \leqq \pi}\left|s_{N, k}(\lambda)\right|<\alpha-\epsilon\right)-\delta \leqq P\left(\max _{0 \leqq \lambda \leqq \pi} \sqrt{N}\left|F_{N, \xi}^{*}(\lambda)-\frac{\lambda}{2 \pi}\right| \leqq \alpha\right) \\
\\
\leqq P\left(\max _{0 \leqq \lambda \leqq \pi}\left|s_{N, k}(\lambda)\right|<\alpha+\epsilon\right)+\delta
\end{aligned}
$$

for $k$ sufficiently large, uniformly in $N>N(\epsilon, \delta)$. But

$$
s_{N, k}(\lambda)=\frac{c_{0}-N}{2 \pi \sqrt{\bar{N}}} \lambda+\frac{1}{\pi} \sum_{1}^{k} \frac{c_{\nu}}{\sqrt{\bar{N}}} \frac{\sin \nu \lambda}{\nu}
$$

and we have shown in Lemma 2 that the joint distribution of $\left(c_{0}-N\right) / \sqrt{N}$, $c_{1} / \sqrt{N}, \cdots, c_{k} / \sqrt{N}$ ( $k$ fixed) converges to the distribution of $k$ independent normal variables with common mean zero and variances $e+2,1,1, \cdots, 1$. Consider the related process

$$
s_{k}(\lambda)=\frac{\gamma_{0}}{2 \pi} \sqrt{e+2} \lambda+\sum_{1}^{k} \frac{\gamma_{\nu}}{\pi} \frac{\sin \nu \lambda}{\nu}
$$

where the $\gamma$ 's are $N(0,1)$ and independent. It is easily seen that

$$
\lim _{N \rightarrow>\infty} P\left(\max _{0 \leqq \lambda \leqq \pi}\left|s_{N, k}(\lambda)\right| \leqq \alpha\right)=P\left(\max _{0 \leqq \lambda \leqq \pi}\left|s_{k}(\lambda)\right| \leqq \alpha\right)
$$

as the relevant point set in $(k+1)$-space is closed [18]. But on letting $k$ tend to infinity $s_{k}(\lambda)$ converges uniformly to

$$
\zeta(\lambda)=\frac{\gamma_{0}}{2 \pi} \sqrt{e+2} \lambda+\sum_{1}^{\infty} \frac{\gamma_{\nu}}{\pi} \frac{\sin \nu \lambda}{\nu}
$$

(see Paley-Wiener [17], p. 148-151). But then

$$
\begin{aligned}
P\left(\max _{0 \leqq \lambda \leqq \pi}|\zeta(\lambda)|<\alpha-\epsilon\right)-\delta<P\left(\max _{0 \leqq \lambda \leqq \pi}\right. & \left.\sqrt{N}\left|F_{N, \xi}^{*}(\lambda)-\frac{\lambda}{2 \pi}\right| \leqq \alpha\right) \\
& <P\left(\max _{0 \leqq \lambda \leqq \pi}|\zeta(\lambda)|<\alpha+\epsilon\right)+\delta
\end{aligned}
$$

and if $N \rightarrow \infty$ we can let $\delta, \epsilon \rightarrow 0$. Since the distribution function of $\max _{0} \leqq \lambda \leqq \pi$ $|\zeta(\lambda)|$ is continuous (see Section 7), this completes the proof.

5. Reduction to pure white noise. Let $G(\lambda)=\int_{0}^{\lambda} f^{2}(l) d l$.

THeORem 4. Let $f(\lambda)$ be a nonnegative absolutely continuous function. Then $\lim _{N \rightarrow \infty} P\left\{\max _{0 \leqq \lambda \leqq \pi} \sqrt{N}\left|\int_{0}^{\lambda} f(l)\left\{2 \pi I_{N, \xi}(l)-1\right\} d l\right| \leqq \alpha\right\}=P\left\{\max _{0 \leqq \lambda \leqq \pi}|\eta(\lambda)|<\alpha\right\}$ 
where $\eta(\lambda)$ is the normal process with, $E_{\eta}(\lambda)=0, E \eta(\lambda) \eta(\mu)=e F(\lambda) F(\mu)$ $+2 \pi G(\min (\lambda, \mu))$.

Proof. Integrating by parts we have

$$
\begin{aligned}
\int_{0}^{\lambda} f(l)\left\{2 \pi I_{N, \xi}(l)-1\right\} d l=2 \pi f(\lambda) & {\left[F_{N, \xi}^{*}(\lambda)-\frac{\lambda}{2 \pi}\right] } \\
& -\int_{0}^{\lambda} 2 \pi f^{\prime}(l)\left[F_{N, \xi}^{*}(l)-\frac{l}{2 \pi}\right] d l .
\end{aligned}
$$

Given $\epsilon>0$, by Theorem 3 we know that

$$
\max _{0 \leqq \lambda \leqq \pi}\left|\sqrt{N}\left(F_{N, \xi}^{*}(\lambda)-\frac{\lambda}{2 \pi}\right)-s_{N, k}(\lambda)\right|<\epsilon
$$

with probability $1-\epsilon$ uniformly in $N>N(\epsilon)$ for $k$ sufficiently large. But then

$$
\begin{aligned}
\max _{0 \leqq \lambda \leqq \pi} \mid f(\lambda)\left[\sqrt{N}\left(F_{N, \xi}^{*}(\lambda)-\frac{\lambda}{2 \pi}\right)-s_{N, k}(\lambda)\right] \\
-\int_{0}^{\lambda} f^{\prime}(l)\left[\sqrt{N}\left(F_{N, \xi}^{*}(l)-\frac{l}{2 \pi}\right)-s_{N, k}(l)\right] d l \mid<B_{1} \epsilon
\end{aligned}
$$

with probability $1-\epsilon$. However,

$$
\begin{aligned}
P\left\{\max _{0 \leqq \lambda \leqq \pi}\left|f(\lambda) s_{N, k}(\lambda)-\int_{0}^{\lambda} f^{\prime}(l) s_{N, k}(l) d l\right| \leqq \alpha\right\} & \\
& \rightarrow P\left\{\max _{0 \leqq \lambda \leqq \pi}\left|f(\lambda) s_{k}(\lambda)-\int_{0}^{\lambda} f^{\prime}(l) s_{k}(l) d l\right| \leqq \alpha\right\}
\end{aligned}
$$

as $N \rightarrow \infty$ as the relevant set is again closed in $(k+1)$-space. We know that for any $\epsilon>0$

$$
\max _{0 \leqq \lambda \leqq \pi}\left|s_{k}(\lambda)-\zeta(\lambda)\right|<\epsilon
$$

with probability $1-\epsilon$ for sufficiently large $k$. Let

$$
\eta(\lambda)=f(\lambda) \zeta(\lambda)-\int_{0}^{\lambda} f^{\prime}(l) \zeta(l) d l
$$

In summary

$$
\begin{aligned}
& P\left\{\max _{0 \leqq \lambda \leqq \pi}|\eta(\lambda)|<\alpha-\epsilon\right\}-\delta<P\left\{\max _{0 \leqq \lambda \leqq \pi} \sqrt{N}\left|\int_{0}^{\lambda} f(l)\left\{2 \pi I_{N, \xi}(l)-1\right\} d l\right| \leqq \alpha\right\} \\
& <P\left\{\max _{0 \leqq \lambda \leqq \pi}|\eta(\lambda)|<\alpha+\epsilon\right\}+\delta
\end{aligned}
$$

and on letting $N \rightarrow \infty$ so that $\epsilon, \delta \rightarrow 0$ we obtain the desired result. Note that the event $\max _{0 \leqq \lambda \leqq \pi}|\eta(\lambda)| \leqq \alpha$ has a well defined probability since $\eta(\lambda)$ is a process with continuous sample functions with probability one. 
Theorem 5. Let $a_{\nu}=O\left(\nu^{\beta}\right)$ where $\beta<-3 / 2$. Then given any $\epsilon>0$

$$
\sqrt{N} \max _{0 \leqq \lambda \leqq \pi}\left|\int_{0}^{\lambda}\left[I_{N}(l)-2 \pi f(l) I_{N, \xi}(l)\right] d l\right|<\epsilon
$$

with probability $1-\epsilon$ for $N>N_{\epsilon}$.

Proof. We have

$$
2 \pi \sqrt{N} \int_{0}^{\lambda}\left[I_{N}(l)-2 \pi f(l) I_{N, \xi}(l)\right] d l=\frac{1}{\sqrt{N}} \sum_{r, s=-\infty}^{\infty} a_{r} a_{s} d_{r s}
$$

where

$$
d_{r, s}=\sum_{n, m=1}^{N} \xi_{n-r} \xi_{m-s} \frac{e^{i(n-m) \lambda}-1}{i(n-m)}-\sum_{\substack{n=1+r, N+r \\ m=1+s, N+s}} \xi_{n-r} \xi_{m-s} \frac{e^{i(n-m) \lambda}-1}{i(n-m)} .
$$

The coefficients of the terms when $n=m$ should be interpreted as $\lambda$. We note that there may be a lattice rectangle $R_{r, s}^{(N)}$ of points $(n, m)$ common to both sums. Let us call the complement of $R_{r, s}^{(N)}$ with respect to the set consisting of all the lattice points in both summations $C_{r, s}^{(N)}$. Then we have

$$
E \max _{0 \leqq \lambda \leqq \pi}\left|d_{r, s}\right| \leqq \sum_{n, m \varepsilon C_{r, s}^{(N)}} g(n-m)
$$

where

$$
g(x)=\left\{\begin{array}{c}
\frac{2}{|x|} \text { if } x \neq 0 \\
\pi \text { if } x=0
\end{array}\right.
$$

It is easily verified that

$$
\sum_{n, m \varepsilon C_{r, s}^{(N)}} g(n-m) \leqq\left\{\begin{array}{l}
2 N \log N \text { if }|r|>N \text { or }|s|>N \\
4(s+r) \log N \text { otherwise. }
\end{array}\right.
$$

The expression in (5.1) is then bounded by

$$
\begin{aligned}
\frac{2}{\sqrt{\bar{N}}} \sum_{s=-\infty}^{\infty} \sum_{|r|>N} \mid & a_{r} a_{s}\left|2 N \log N+\frac{8}{\sqrt{\bar{N}}} \sum_{s=-\infty}^{\infty} \sum_{|r| \leqq N}\right| a_{r} a_{s} \mid r \log N \\
& \leqq\left(\sum_{s=-\infty}^{\infty}\left|a_{s}\right|\right)\left(4 \sqrt{N} \log N \sum_{|r|>N}\left|a_{r}\right|+\frac{8 \log N}{\sqrt{\bar{N}}} \sum_{|r| \leqq N} r\left|a_{r}\right|\right) .
\end{aligned}
$$

Under the assumption made above, this expression tends to zero as $N \rightarrow \infty$ which proves the theorem.

6. Treatment of the general case. Let $F_{N}^{*}(\lambda)=\int_{0}^{\lambda} I_{N}(l) d l$.

Theorem 6. Let

1. $f(\lambda)$ be absolutely continuous

2. $a_{\nu}=0\left(\nu^{\beta}\right), \beta<-3 / 2$. 
Then

$$
\lim _{v \rightarrow \infty} P\left\{\max _{0 \leqq \lambda \leqq \pi} \sqrt{N}\left|F_{N}^{*}(\lambda)-F(\lambda)\right| \leqq \alpha\right\}=P\left\{\max _{0 \leqq \lambda \leqq \pi}|\eta(\lambda)| \leqq \alpha\right\}
$$

where $\eta(\lambda)$ is the process defined in Section 5.

The proof follows immediately from Theorems 3, 4 and 5. An important special case is contained in the following corollary.

Corollary 1. When $y_{t}$ is a normal process with a positive spectral density with an integrable second derivative, Theorem 6 reduces to the following statement:

$$
\begin{aligned}
\lim _{N \rightarrow \infty} P\left\{\max _{0 \leqq \lambda \leqq \pi}\right. & \left.\sqrt{N}\left|F_{N}^{*}(\lambda)-F(\lambda)\right| \leqq \alpha\right\} \\
& =\sum_{k=-\infty}^{\infty}(-1)^{k}[\Phi((2 k+1) \alpha / x)-\Phi((2 k-1) \alpha / x)]=\Delta(\alpha / x)
\end{aligned}
$$

where $\Phi(u)$ is the normal distribution function and $x=\sqrt{2 \pi G(\pi)}$.

Proof. Lemma 3 implies that the assumptions of Theorem 6 are satisfied.

As $e=0$ the process $\eta(\lambda)$ reduces to the Wiener process with the following changed scale of time: $t=2 \pi G(\lambda), 0 \leqq \lambda \leqq \pi$. The reader may note that (6.1) is the probability that a particle in Brownian motion on the line is not absorbed by the barriers $\alpha,-\alpha$ in the time interval $0,2 \pi G(\pi)$, given that the particle starts from 0 at time 0 (see Sommerfeld [19], pp. 74-79).

7. The limiting distribution in the case of pure white noise. When the process $y_{t}$ is pure white noise (so that $f(\lambda) \equiv$ constant, say 1 ) the limiting probability we are interested in is given by

$$
P\left\{\max _{0 \leqq \lambda \leqq \pi}|\zeta(\lambda)| \leqq \alpha\right\}
$$

where $\zeta(\lambda)$ is the normal process with

$$
\begin{aligned}
E \zeta(\lambda) & =0 \\
E \zeta(\lambda) \zeta(\mu) & =e \lambda \mu+2 \pi \min (\lambda, \mu) .
\end{aligned}
$$

Now (7.1) has been explicitly evaluated when $e=0$ (see 6.1). However, the limiting distribution can still be evaluated when $e \neq 0$.

TheOREM 7.

$$
\begin{aligned}
& P\left\{\max _{0 \leqq \lambda \leqq \pi}|\zeta(\lambda)| \leqq \alpha\right\}=\sum_{k=-\infty}^{\infty}(-1)^{k} e^{\left(-k^{2} \alpha^{2} / \pi^{2}\right)(1-(\gamma / 2))} \\
& \cdot\left[\Phi\left(\frac{\sqrt{\gamma} \alpha}{\pi}\left(k+\frac{1}{\gamma}\right)-\Phi\left(\frac{\sqrt{\gamma} \alpha}{\pi}\left(k-\frac{1}{\gamma}\right)\right)\right]\right.
\end{aligned}
$$

where $\gamma=e+2$.

Proof. Let $\tau=\lambda / \pi$. Let $X$ be a normal random variable with mean zero and variance one. Let $X(\tau)$ be a normal process with

$$
\begin{aligned}
E X(\tau) & =0 \\
E X(\tau) X\left(\tau^{\prime}\right) & =\min \left(\tau, \tau^{\prime}\right)-\tau \tau^{\prime},
\end{aligned}
$$


that is, $X(\tau)$ is the Wiener process conditioned so that $X(1)=0 . X$ and $X(\tau)$ are independent. Consider the process $u(\tau)=\sqrt{\gamma} \pi \tau X+\pi \sqrt{2} X(\tau)$. The process $u(\tau)$ has the same probability distribution as $\zeta(\lambda)$ so that

But

$$
P\left\{\max _{0 \leqq \lambda \leqq \pi}|\zeta(\lambda)| \leqq \alpha\right\}=P\left\{\max _{0 \leqq \tau \leqq 1}|u(\tau)| \leqq \alpha\right\} .
$$

$$
\begin{aligned}
& P\left\{\max _{0 \leqq \tau 1}|u(\tau)| \leqq \alpha\right\} \\
& \quad=\int_{-\infty}^{\infty} P\left\{\frac{-\alpha-\sqrt{\gamma} \pi \tau x}{\pi \sqrt{2}} \leqq X(\tau) \leqq \frac{\alpha-\sqrt{\gamma} \pi \tau x}{\pi \sqrt{2}} ; 0 \leqq \tau \leqq 1\right\} \varphi(x) d x
\end{aligned}
$$

where $\varphi(x)$ is the normal frequency function. Let

$$
Y(t)=(t+1) X\left(\frac{t}{t+1}\right), \quad 0 \leqq t<\infty .
$$

Then $Y(t)$ is the Wiener process (see [4]), $0 \leqq t<\infty$. We have then to consider

$$
P\{-a-(a+b) t \leqq y(t) \leqq a+(a-b) t ; 0 \leqq t<\infty\}
$$

where $a=\alpha / \pi \sqrt{2}, b=\sqrt{\gamma} x / \sqrt{2}$. The integrand in (7.2) is clearly zero unless $|\sqrt{\gamma} x| \leqq \alpha / \pi$. But supposing this inequality to hold, Doob [4] has evaluated (7.3) as

$$
\begin{aligned}
\sum_{m=1}^{\infty}\left\{e^{-2\left[(2 m-1) 2_{a}^{2}-(2 m-1) b a\right]}+e^{-2\left[(2 m-1) 2_{a}^{2}+(2 m-1) b a\right]}\right. & \left.-e^{-2\left[(2 m)^{2} a^{2}-2 m b a\right]}-e^{-2\left[(2 m){ }^{2} a^{2}+2 m b a\right]}\right\} \\
& =2 \sum_{n=1}^{\infty}(-1)^{n+1} e^{-2 n^{2} a^{2}} \cosh 2 n b a .
\end{aligned}
$$

Thus (7.1) is equal to

$$
\begin{array}{r}
\Phi\left(\frac{\alpha}{\pi \sqrt{\gamma}}\right)-\Phi\left(\frac{-\alpha}{\pi \sqrt{\gamma}}\right)-2 \sum_{n=1}^{\infty}(-1)^{n+1} e^{-\left(n^{2} \alpha^{2} / \pi^{2}\right)} \int_{-\alpha / \pi \sqrt{\gamma}}^{\alpha / \pi \sqrt{\gamma}} \varphi(x) \cosh n \frac{\sqrt{\gamma} \alpha}{\pi} x d x \\
=\Phi\left(\frac{\alpha}{\pi \sqrt{\gamma}}\right)-\Phi\left(\frac{-\alpha}{\pi \sqrt{\gamma}}\right)-\sum_{n=1}^{\infty}(-1)^{n-1} e^{-\left(n^{2}\left[\left(\alpha^{2} / \pi^{2}\right)-\left(\alpha^{2} / 2 \pi^{2}\right)\right]\right)} \\
\left\{\Phi\left(\frac{n \sqrt{\gamma} \alpha}{\pi}+\frac{\alpha}{\pi \sqrt{\gamma}}\right)-\Phi\left(\frac{n \sqrt{\gamma} \alpha}{\pi}-\frac{\alpha}{\pi \sqrt{\gamma}}\right)+\Phi\left(\frac{-n \sqrt{\gamma} \alpha}{\pi}+\frac{\alpha}{\pi \sqrt{\gamma}}\right)\right. \\
\left.-\Phi\left(-\frac{n \sqrt{\gamma} \alpha}{\pi}-\frac{\alpha}{\pi \sqrt{\gamma}}\right)\right\}=\sum_{n=-\infty}^{\infty}(-1)^{n} e^{-\left(n^{2} \alpha^{2} / \pi^{2}\right)(1-(\gamma / 2))} \\
\cdot\left[\Phi\left(\frac{\sqrt{\gamma} \alpha}{\pi}\left(n+\frac{1}{\gamma}\right)\right)-\Phi\left(\frac{\sqrt{\gamma} \alpha}{\pi}\left(n-\frac{1}{\gamma}\right)\right)\right] .
\end{array}
$$

This expression parallels (6.1) and will be used in a later publication in which we will study some applications of the theory.

8. Statistical applications. The corollary of Theorem 6 lends itself to important statistical applications. First, however, we will have to estimate $G(\pi)$ which 
is in general unknown. We note that $G(\pi)=(4 \pi)^{-1} \sum_{-\infty}^{\infty} \rho_{\nu}^{2}$. Of course the $C_{\nu} / N$ are consistent estimates of $\rho_{\nu}$. It is natural to consider an estimate of the form (8.1). One reason for this particular choice is that in practice one will try to avoid the cumbersome calculation of all the lagged product sums $C_{\nu}$.

Lemma 4. If $a_{\nu}=0\left(\nu^{\beta}\right), \beta<-1$, the statistic

$$
G^{*}(\pi)=\frac{1}{4 \pi N^{2}}\left[C_{0}^{2}+2 \sum_{\nu=1}^{\left[K N^{\alpha}\right]} C_{\nu}^{2}\right], \quad 0<\alpha<1, \quad K>0,
$$

is a consistent estimate of $G(\pi)$.

Proof.

$$
G^{*}(\pi)=\frac{1}{4 \pi N^{2}}\left\{C_{0}^{2}+2 \sum_{\nu=1}^{m} C_{\nu}^{2}\right\}+\frac{1}{2 \pi N^{2}} \sum_{\nu=m+1}^{\left[R N^{\alpha \alpha}\right]} C_{\nu}^{2} .
$$

But we see using (3.5) that the last sum is less than or equal to

$$
\begin{aligned}
& \sum_{\nu=m+1}^{\left[K N^{\alpha}\right]} \sum_{j, k=1}^{N} \sum_{\beta_{1} \beta_{2}=-\infty}^{\infty}\left\{\left|a_{j-\beta_{1}} a_{j+\nu-\beta_{1}} a_{k-\beta_{2}} a_{k+\nu-\beta_{2}}\right|+\left|a_{j-\beta_{1}} a_{j+\nu-\beta_{2}} a_{k-\beta_{1}} a_{k+\nu-\beta_{2}}\right|\right. \\
& \left.\quad+\left|a_{j-\beta_{1}} a_{j+\nu-\beta_{2}} a_{k-\beta_{2}} a_{k+\nu-\beta_{1}}\right|\right\}\left(4+\mu_{4}\right)=\left(\sum_{1}+\sum_{2}+\sum_{3}\right)\left(4+\mu_{4}\right)^{.}
\end{aligned}
$$

Let the $r_{\nu}$ be understood to be the covariances of the process $\sum_{\nu=-\infty}^{\infty}\left|a_{n-\nu}\right| \xi_{\nu}$. The spectral density $(2 \pi)^{-1}\left|\sum\right| a_{\nu}\left|e^{i \nu \lambda}\right|^{2}$ of this process is continuous and hence quadratically integrable, so that $\sum_{-\infty}^{\infty} r_{\nu}^{2}<\infty$. But then

$$
\left|\sum_{1}\right|=\sum_{\nu=m+1}^{\left[K N^{\alpha}\right]} N^{2} r_{\nu}^{2} \leqq N^{2} \sum_{\nu=m+1}^{\infty} r_{\nu}^{2}
$$

so that $\left|\sum_{1}\right| / N^{2}<\epsilon$ for $m$ sufficiently large uniformly in $N$. Also we get

$$
\frac{1}{N^{2}}\left|\sum_{2}\right|=\frac{1}{N^{2}} \sum_{\nu=m+1}^{\left[K N^{\alpha}\right]} \sum_{j, k=1}^{N} r_{j-k}^{2} \leqq \frac{1}{N} \sum_{m+1}^{\left[K N^{\alpha}\right]} \sum_{n=-\infty}^{\infty} r_{n}^{2} \leqq \frac{K N^{\alpha}+1}{N} \sum_{-\infty}^{\infty} r_{n}^{2} \rightarrow 0
$$

as $N \rightarrow \infty$. The third sum is handled in almost the same way but using Schwarz' inequality. We now choose $m$ so large that the last sum in (8.2) is less than $\delta$ with probability larger than $1-\delta$. The term in brackets in (8.2) consists of a fixed number of terms each of which converges in probability to the corresponding term in the expression for $G(\pi)$. This proves the lemma.

The corollary of Theorem 6 shows that in the normal domain the asymptotic distribution of $\max _{\lambda} \sqrt{N}\left|F_{N}^{*}(\lambda)-F(\lambda)\right|$ depends only on one parameter $G(\pi)$. This together with Lemma 4 enables us to construct confidence bands for $F(\lambda)$.

Theorem 8. Suppose that $y_{t}$ is a normal process and that its spectral density is positive and has an integrable second derivative. Then

$$
F_{N}^{*}(\lambda)-\alpha \sqrt{\frac{2 \pi G^{*}(\pi)}{N}} \leqq F(\lambda) \leqq F_{N}^{*}(\lambda)+\alpha \sqrt{\frac{2 \pi G^{*}(\pi)}{N}}
$$

holds with a probability tending to $\Delta(\alpha)$ as $N$ tends to infinity.

The proof follows immediately from Lemma 4 and Corollary 1 as $\Delta(\alpha)$ is continuous.

Clearly Theorem 8 also gives a test of significance for the simple hypothesis 
of a completely specified spectrum. A more interesting case, however, is the following.

Theorem 9. From a stochastic process satisfying Theorem 8 we observe two independent time series of length $N_{1}$ and $N_{2}$. Compute the corresponding estimates $F_{N_{1}}^{*}(\lambda), F_{N_{2}}^{*}(\lambda), G_{1}^{*}(\pi), G_{2}^{*}(\pi)$. Then, assuming that $N_{1} / N_{2} \rightarrow c>0$ and putting $N=2 N_{1} N_{2} /\left(N_{1}+N_{2}\right)$,

$$
\frac{\max _{0 \leqq \lambda \leqq \pi} \sqrt{N}\left|F_{N_{1}}^{*}(\lambda)-F_{N_{2}}^{*}(\lambda)\right|}{\sqrt{G_{1}^{*}(\pi)+G_{2}^{*}(\pi)}}>\alpha,
$$

holds with a probability tending to $1-\Delta(\alpha)$ as $N$ tends to infinity under the hypothesis $F_{1}(\lambda)=F_{2}(\lambda)$.

Theorems 8 and 9 are somewhat restricted in their applicability as they stand, since in many contexts $m_{t}=E x_{t}$ is not identically zero. We shall consider the case

$$
m_{t}=d_{1} \varphi_{t}^{(1)}+d_{2} \varphi_{t}^{(2)}+\cdots+d_{p} \varphi_{t}^{(p)},
$$

where $\varphi_{t}^{(1)}, \varphi_{t}^{(2)}, \cdots, \varphi_{t}^{(p)}$ are given sequences and the regression coefficients $d_{\nu}$ are unknown. To avoid unnecessary complications we will confine ourselves to the case $p=2$, which illustrates the general situation. We have to introduce the following condition which prevents the two regression variables $\varphi_{t}^{(1)}$ and $\varphi_{t}^{(2)}$ from becoming linearly dependent in the limit

$$
R=\varlimsup_{N \rightarrow \infty} \frac{\left(\sum_{1}^{N} \varphi_{t}^{(1)} \varphi_{t}^{(2)}\right)^{2}}{\sum_{1}^{N} \varphi_{t}^{(1)^{2}} \sum_{1}^{N} \varphi_{t}^{(2)^{2}}}<1 .
$$

We will use the least square estimates $d_{1}^{*}, d_{2}^{*}$ of $d_{1}, d_{2}$ as they have been shown to be asymptotically efficient [8].

Theorem 10. Under the conditions of Theorems 8 and 9 and (8.5), formulas (8.3) and (8.4) remain valid if $F_{N}^{*}(\lambda)$ is computed using $x_{t}-d_{1}^{*} \varphi_{t}^{(1)}-d_{2}^{*} \varphi_{t}^{(2)}$ in place of $y_{t}$.

Proof. It is sufficient to prove that

$$
\begin{aligned}
\max _{0 \leqq \lambda \leqq \pi} \sqrt{N}\left|\frac{1}{2 \pi N}\right|_{0}^{\lambda} \mid \sum_{\nu=1}^{N}\left(x_{\nu}-d_{1}^{*} \varphi_{\nu}^{(1)}\right. & \left.-d_{2}^{*} \varphi_{\nu}^{(2)}\right)\left.e^{-i \nu l}\right|^{2} d l \\
& -\frac{1}{2 \pi N} \int_{0}^{\lambda}\left|\sum_{1}^{N} y_{\nu} e^{-i \nu l}\right|^{2} d l
\end{aligned}
$$

tends to zero in probability as $N \rightarrow \infty$. The expression inside the absolute value sign is

$$
\begin{aligned}
& \frac{1}{2 \pi N} \sum_{\nu, \mu=1}^{N}\left[-2\left(d_{1}^{*}-d_{1}\right) \varphi_{\nu}^{(1)} y_{\mu}-2\left(d_{2}^{*}-d_{2}\right) \varphi_{\nu}^{(2)} y_{\mu}+\left(d_{1}^{*}-d_{1}\right)^{2} \varphi_{\nu}^{(1)} \varphi_{\mu}^{(1)}\right. \\
& \left.+\left(d_{2}^{*}-d_{2}\right)^{2} \varphi_{\nu}^{(2)} \varphi_{\mu}^{(2)}+2\left(d_{1}^{*}-d_{1}\right)\left(d_{2}^{*}-d_{2}\right) \varphi_{\nu}^{(1)} \varphi_{\mu}^{(2)}\right] \cdot \frac{\sin (\nu-\mu) \lambda}{\nu-\mu} \\
& =\sum_{1}+\sum_{2}+\sum_{3}+\sum_{4}+\sum_{5}
\end{aligned}
$$


where the same convention as before is used in interpreting the terms with $\nu=\mu$. The least square estimates $d_{1}^{*}, d_{2}^{*}$ are unbiased linear estimates with coefficients depending only upon the $\varphi_{\nu}^{(1)}$ s $\mathrm{s}$ and $\varphi_{\nu}^{(2)}$ 's. A simple argument using the fact that the spectral density is bounded shows that

(8.6) $\quad \operatorname{var} d_{1}^{*} \leqq 2 \pi \max _{0 \leqq \lambda \leqq \pi} f(\lambda) \frac{\sum_{\nu} \varphi_{\nu}^{(2)}}{\sum \varphi_{\nu}^{(1)^{2}} \sum \varphi_{\nu}^{(2)}-\left(\sum \varphi_{\nu}^{(1)} \varphi_{\nu}^{(2)}\right)^{2}}$

$\leqq \frac{2 \pi \max _{0 \leqq \lambda \leqq \pi} f(\lambda)}{1-R} \frac{1}{\sum_{1}^{N} \varphi_{\nu}^{(1) 2}}$.

Now

$$
\begin{aligned}
& \pi \sqrt{\bar{N}} \max _{0 \leqq \lambda \leqq \pi}\left|\sum_{1}\right| \leqq \frac{1}{\sqrt{N}}\left\{\left|d_{1}^{*}-d_{1}\right| \sum_{p=1}^{N=1} \frac{1}{p}\left|\sum_{\nu=1}^{N-p} y_{\nu} \varphi_{\nu+p}^{(1)}\right|\right. \\
& \left.\quad+\pi\left|d_{1}^{*}-d_{1}\right| \cdot\left|\sum_{\nu=1}^{N} y_{\nu} \varphi_{\nu}^{(1)}\right|^{2}+\left|d_{1}^{*}-d_{1}\right| \sum_{p=-N+1}^{1}\left|\frac{1}{p}\right| \sum_{\nu=1-p}^{N} y_{\nu} \varphi_{\nu+p}^{(1)} \mid\right\} .
\end{aligned}
$$

But we have

$$
E\left[\sum_{\nu=1}^{N-p} y_{\nu} \varphi_{\nu+p}^{(1)}\right]^{2} \leqq 2 \pi \max _{0 \leqq \lambda \leqq \pi} f(\lambda) \sum_{\nu=1}^{N} \varphi_{\nu}^{(1)^{2}}
$$

We know from (8.6) that with as large a probability as desired $\left|d_{1}^{*}-d_{1}\right|$ is less than $k / \sqrt{\sum_{v=1}^{N} \varphi_{\nu}^{(2)}}$. Then it follows from Schwarz' inequality and (8.7) that with large probability

$$
\pi \sqrt{\bar{N}} \max _{0 \leqq \lambda \leqq \pi}\left|\sum_{1}\right|<K^{\prime} \log N / \sqrt{N} \rightarrow 0 .
$$

$\sum_{2}$ can be handled in the same way. Now

$$
2 \pi \sqrt{\bar{N}} \max _{0 \leqq \lambda \leqq \pi}\left|\sum_{3}\right|=\left.\frac{\left(d_{1}^{*}-d_{1}\right)^{2}}{\sqrt{\bar{N}}}\right|_{0} ^{\lambda}\left|\sum_{1}^{N} \varphi_{\nu}^{(1)} e^{i \nu l}\right|^{2} d l \leqq \frac{\left(d_{1}^{*}-d_{1}\right)^{2}}{\sqrt{\bar{N}}} \pi \sum_{1}^{N} \varphi_{\nu}^{(1) 2}
$$

and the expectation of this tends to zero as $N^{-\frac{1}{2}} \cdot \sum_{4}$ and $\sum_{5}$ can be treated in a similar manner which proves the theorem.

An important special case is $\varphi_{\nu} \equiv 1$, which corresponds to a constant unknown mean value of the process. Another situation of some interest arises when the spectrum of the process has a discrete component with frequencies $\lambda_{1}, \lambda_{2}, \cdots, \lambda_{p}$. There we take the $\varphi_{\nu}$ 's as trigonometric functions with these frequencies.

9. Alternative estimates of the spectral distribution function. It is clear that Theorems 6 , and 8-10 are still valid if the estimate $F_{N}^{*}(\lambda)$ is replaced by a truncated estimate

$$
F_{N, t r}^{*}(\lambda)=\frac{C_{0} \lambda}{2 \pi N}+\frac{1}{\pi N} \sum_{\nu=1}^{h_{N}} C_{\nu} \frac{\sin \nu \lambda}{\nu}=\frac{1}{2 \pi} \int_{0}^{\lambda} \int_{-\pi}^{\pi} I_{N}(l) \frac{\sin \left(h_{N}+\frac{1}{2}\right)(l-\mu)}{\sin (l-\mu / 2)} d l d \mu
$$


where $h_{N} \rightarrow \infty$ as $N \rightarrow \infty$, as they can be proved in exactly the same manner since the weighting factors are 1 from $\nu=0$ up to $\nu=h_{N}$ and 0 from that point on. Note that the estimate $F_{N, t r}^{*}(\lambda)$, in general, is not nondecreasing with probability 1 which may at times be an unpleasant feature. We can choose $h_{N}=\left[k N^{\alpha}\right]$ as in Lemma 4 . This reduces the computational work considerably as one then only needs to compute the $C_{\nu}$ 's for $\nu \leqq\left[k N^{\alpha}\right]$.

We shall now consider a class of estimates of the spectral distribution function with nondecreasing weight functions. Let $W_{N}(\lambda)=\int_{-\pi}^{\lambda} w_{N}(l) d l$ where $w_{N}(l) \geqq 0$ in $(-\pi, \pi), \int_{-\pi}^{\pi} w_{N}(l) d l=1$ and

Let

$$
\lim _{N \rightarrow \infty} W_{N}(\lambda)=\left\{\begin{array}{lll}
0 & \text { if } & \lambda<0 \\
1 & \text { if } & \lambda>0 .
\end{array}\right.
$$

$$
\begin{gathered}
F_{N}^{*}\left(\lambda, W_{N}\right)=\int_{0}^{\pi} I_{N}(l) W_{N}(\lambda-l) d l \\
F\left(\lambda, W_{N}\right)=\int_{0}^{\pi} f(l) W_{N}(\lambda-l) d l .
\end{gathered}
$$

We prove the following theorem.

Theorem 11. Under the conditions of Theorem 6

$\lim _{N \rightarrow \infty} P\left\{\max _{0 \leqq \lambda \leqq \pi} \sqrt{N}\left|F_{N}^{*}\left(\lambda, W_{N}\right)-F\left(\lambda, W_{N}\right)\right| \leqq \alpha\right\}=P\left\{\max _{0 \leqq \lambda \leqq \pi}|\eta(\lambda)| \leqq \alpha\right\}$.

Proof. On integrating by parts we have

$$
\begin{aligned}
& \sqrt{N}\left[F_{N}^{*}\left(\lambda, W_{N}\right)-F\left(\lambda, W_{N}\right)\right]=\sqrt{N} \int_{0}^{\pi}\left[I_{N}(l)-f(l)\right] d l W_{N}(\lambda-\pi) \\
&+\int_{0}^{\pi} \sqrt{N} \int_{0}^{l}\left[I_{N}(\mu)-f(\mu)\right] d \mu w_{N}(\lambda-l) d l .
\end{aligned}
$$

By Theorem 5 we can replace (9.1) by

$$
\begin{aligned}
\sqrt{N} \int_{0}^{\pi} f(l)\left[2 \pi I_{N, \xi}(l)\right. & -1] d l W_{N}(\lambda-\pi) \\
& +\int_{0}^{\pi} \sqrt{N} \int_{0}^{l}\left[2 \pi I_{N, \xi}(\mu)-1\right] f(\mu) d \mu w_{N}(\lambda-l) d l
\end{aligned}
$$

committing an error of at most $\epsilon>0$ uniformly in $\lambda$ with probability $1-\epsilon$. However, on integrating by parts twice we obtain

$$
\begin{array}{r}
\sqrt{N} 2 \pi\left[F_{N, \xi}^{*}(\pi)-\frac{1}{2}\right] f(\pi) W_{N}(\lambda-\pi)-\int_{.0}^{\pi} \sqrt{N} 2 \pi\left[F_{N, \xi}^{*}(l)-\frac{l}{2 \pi}\right] \\
\cdot \frac{d}{d l}\left(f(l) W_{N}(\lambda-l)\right) d l .
\end{array}
$$


But

$$
\sqrt{\bar{N}} \max _{0 \leqq \lambda \leqq \pi}\left|F_{N, \xi}^{*}(\lambda)-\frac{\lambda}{2 \pi}-\frac{1}{\sqrt{\bar{N}}} s_{N, m}(\lambda)\right|<\epsilon
$$

with probability $1-\epsilon$ where $m$ is a large but fixed number. We can then replace (9.3) by

$$
\begin{aligned}
\int_{0}^{\pi} f(l) W_{N}(\lambda-l) s_{N, m}^{\prime}(l) d l=\frac{c_{0}-N}{2 \pi \sqrt{N}} \int_{0}^{\pi} f(l) W_{N}(\lambda-l) d l \\
\quad+\frac{1}{\pi} \sum_{1}^{m} \int_{0}^{\pi} \frac{c_{\nu}}{\sqrt{\bar{N}}} \cos \nu l f(l) W_{N}(\lambda-l) d l
\end{aligned}
$$

with an error of at most $\epsilon$ uniformly in $\lambda$. But then reasoning as in Theorem 4 we get

$$
\begin{aligned}
& \lim _{N \rightarrow \infty} P\left\{\max _{0 \leqq \lambda \leqq \pi}\left|\int_{0}^{\pi} f(l) W_{N}(\lambda-l) s_{N, m}^{\prime}(l) d l\right| \leqq \alpha\right\} \\
& =P\left\{\max _{0 \leqq \lambda \leqq \pi}\left|\int_{0}^{\lambda} f(l) s_{m}^{\prime}(l) d l\right| \leqq \alpha\right\}
\end{aligned}
$$

making use of the fact that

$$
\begin{aligned}
\max _{0 \leqq \lambda \leqq \pi} \mid \int_{0}^{\pi} \cos \nu l f(l) W_{N}(\lambda & -l) d l-\int_{0}^{\lambda} \cos \nu l f(l) d l \mid \\
\leqq & \max _{0 \leqq \lambda \leqq \pi} f(\lambda)\left\{\int_{-\pi}^{0} W_{N}(l) d l+\int_{0}^{\pi}\left|W_{N}(l)-1\right| d l\right\} \rightarrow 0
\end{aligned}
$$

as $N \rightarrow \infty$. But

$$
\max _{0 \leqq \lambda \leqq \pi}\left|\int_{0}^{\lambda} f(l) s_{m}^{\prime}(l) d l-\eta(\lambda)\right|<\epsilon
$$

with large probability if $m$ is sufficiently large and the theorem follows immediately.

We are usually more interested in estimating $F(\lambda)$ than $F\left(\lambda, W_{N}\right)$. The following corollary enables us to do this.

Corollary 2. Theorems $6,8-10$ remain valid when $F_{N}^{*}(\lambda)$ is replaced by $F_{N}^{*}\left(\lambda, W_{N}\right)$ if

$$
\int_{-\pi}^{0} W_{N}(\lambda) d \lambda+\int_{0}^{\pi}\left[1-W_{N}(\lambda)\right] d \lambda=o\left(N^{-\frac{1}{2}}\right) .
$$

Proof. The proof follows immediately as

$$
\begin{aligned}
& \max _{0 \leqq \lambda \leqq \pi} \sqrt{N}\left|F(\lambda)-F\left(\lambda, W_{N}\right)\right| \\
& \leqq \sqrt{N} \max _{0 \leqq \lambda \leqq \pi} f(\lambda)\left\{\int_{-\pi}^{0} W_{N}(\lambda) d \lambda+\int_{0}^{\pi}\left[1-W_{N}(\lambda)\right] d \lambda\right\}=o(1)
\end{aligned}
$$


It is clear from the comment above that the time series analyst has a large class of estimates of the spectral distribution function at his disposal. At present we are not able to claim that certain estimates are preferable to others. We hope to investigate such problems in a later paper.

It is worthwhile noting that the Fejer kernel

$$
\frac{1}{2 \pi h_{N}} \frac{\sin ^{2} \frac{h_{N}}{2} \lambda}{\sin ^{2} \frac{\lambda}{2}}=w_{N}(\lambda)
$$

satisfies (9.4) if $\log h_{N} / h_{N}=o\left(N^{-\frac{1}{2}}\right)$, so that we can choose the truncation point $h_{N}$ as $h_{N}=\left[k N^{\alpha}\right], \frac{1}{2}<\alpha<1$. Note that the estimate corresponding to this kernel is

$$
F_{N}^{*}\left(\lambda, W_{N}\right)=\frac{C_{0}}{2 \pi N}+\frac{1}{\pi N} \sum_{\nu=1}^{h_{N}} C_{\nu} \frac{\sin \nu \lambda}{\nu}\left(1-\frac{\nu}{h_{N}}\right) .
$$

This estimate of the spectral distribution function is closely related to an estimate of the spectral density given by Bartlett [1]. It is nondecreasing and does not require the computation of all the $C_{\nu}$ 's.

10. Alternative statistics and the corresponding limit theorems. In some instances one might prefer to consider statistics other than $\max _{0 \leqq \lambda \leqq \pi}\left|F_{N}^{*}(\lambda)-F(\lambda)\right|$ in analyzing time series. We shall therefore consider some alternative statistics in this section.

Consider the linear space consisting of continuous functions $c=c(\lambda)$ on $0 \leqq \lambda \leqq \pi$ with the norm $\|c\|=\sup _{0 \leqq \lambda \leqq \pi}|c(\lambda)|$. Consider a functional $\varphi(c)$ uniformly continuous in this topology.

Theorem 12. Under the conditions of Theorem 6 we have

$\lim _{N \rightarrow \infty} P\left\{\varphi\left(\sqrt{N}\left[F_{N}^{*}(\lambda)-F(\lambda)\right]\right) \leqq \alpha, 0 \leqq \lambda \leqq \pi\right\}=P\{\varphi(\eta(\lambda)) \leqq \alpha, 0 \leqq \lambda \leqq \pi\}$.

Proof. Writing

$$
\sqrt{\bar{N}}\left[F_{N}^{*}(\lambda)-F(\lambda)\right]=s_{N, k}(\lambda)+r_{N, k}(\lambda)
$$

as before we note that $\left\|r_{N, k}(\lambda)\right\|<\epsilon$ if $k$ is chosen sufficiently large. Hence we commit only a small error by considering instead the probability of the event

$$
\left\{\varphi\left(s_{N, k}(\lambda)\right) \leqq \alpha, 0 \leqq \lambda \leqq \pi\right\}
$$

which is a closed set in $(k+1)$-space. This probability converges to

$$
P\left\{\varphi\left(s_{k}(\lambda)\right) \leqq \alpha, 0 \leqq \lambda \leqq \pi\right\}
$$

as $N \rightarrow \infty$. But we can choose $k$ so large that $\left\|s_{k}(\lambda)-\eta(\lambda)\right\|<\delta$ with probability $1-\delta$. This together with the uniform continuity of $\varphi(c)$ proves the theorem.

As an example we can choose

$$
\varphi(c)=\left\{\int_{0}^{\lambda}|c(\lambda)|^{p} d \mu(\lambda)\right\}^{1 / p}, \quad p \geqq 1
$$


where $\mu(\lambda)$ is bounded and nondecreasing. This will give a statistic of the von Mises type.

11. Acknowledgement. We are indebted to J. L. Doob who suggested the problem that led to the investigation resulting in this paper.

\section{REFERENCES}

[1] M. S. BARTLETT, "Smoothing periodograms from time series with continuous spectra," Nature, Vol. 161 (1948), pp. 686-687.

[2] M. S. Bartletr, "Periodogram analysis and continuous spectra," Biometrika, Vol. 37 (1950), pp. 1-16.

[3] H. Cramér, "On harmonic analysis in certain functional spaces," Ark. Mat. Astr. Fys., Vol. 28B (1942), p. 12.

[4] J. L. Dоoв, "Heuristic approach to the Kolmogorov-Smirnov theorems," Ann. Math. Stat., Vol. 20 (1949), pp. 393-403.

[5] L. FEJER, "Lebesguesche konstanten und divergente fourier-reihen," J. Reine Angew. Math., Vol. 138 (1910), pp. 22-53.

[6] R. A. Fisher, "Tests of significance in harmonic analysis," Proc. Roy. Soc. London, Ser. A, Vol. 125 (1929), pp. 5t-59.

[7] U. Grenander, "On empirical spectral analysis of stochastic processes," Ark. Mat., Vol. 1 (1951), pp. 503-531.

[8] U. Grenander, "On the estimation of regression coefficients in the case of an autocorrelated disturbance," unpublished manuscript.

[9] U. Grenander and M. Rosenblatt, "On spectral analysis of stationary time series," Proc. Nat. Acad. Sci., U. S. A., Vol. 38 (1952), pp. 519-521.

[10] G. Herglotz, "Ưber Potenzreihen mit positivem reelem Teil im Einheitskreis," Sitzgsber. Sächs. Akad. Wiss., Vol. 63 (1911), p. 501.

[11] W. Hoeffing and H. Robbins, "The central limit theorem for dependent random variables," Duke Math. J., Vol. 15 (1948), pp. 773-780.

[12] K. Karhunen, "Uber lineare Methoden in der Wahrscheinlichkeitsrechnung," Ann. Acad. Sc. Fenn. Ser. A I, Math.-Phys. No. 37, 79 pp. 1947.

[13] M. G. Kendall, The Advanced Theory of Statistics, Vol. 2, C. Griffith and Co., London, 1946.

[14] T. Koopmans, Statistical Inference in Dynamic Economic Models, John Wiley and Sons, 1950.

[15] P. LEvy, Théorie de l'Addition des Variables Aléatoires, Gauthier-Villars, Paris, 1937.

[16] P. LEvy, Les Processus Stochastiques et le Mouvement Brownien, Gauthier-Villars, Paris, 1948.

[17] R. E. A. C. Paley and N. Wiener, Fourier Transforms in the Complex Domain, Amer. Math. Soc., New York, 1934.

[18] H. SCheffe, "A useful convergence theorem for probability distributions," Ann. Math. Stat., Vol. 18 (1947), pp. 434-438.

[19] A. Sommerfeld, Partial Differential Equations in Physics, Academic Press, New York, 1949.

[20] J. TukeY, "Measuring noise color," unpublished manuscript.

[21] P. Whitrte, Hypothesis Testing in Time Series Analysis, Uppsala, 1951.

[22] H. Wold, A Study in the Analysis of Stationary Time Series, Almqvist and Wiksells boktr., Uppsala, 1938. 